\title{
Utilizing nuclear data in delayed gamma-ray spectroscopy inverse Monte Carlo analysis
}

\author{
Douglas Chase Rodriguez ${ }^{1, *}$, Kamel Abbas ${ }^{2}$, Jean-Michel Crochemore ${ }^{2}$, Mitsuo Koizumi ${ }^{1}$, Stefan Nonneman $^{2}$, Bent \\ Pedersen $^{2}$, Fabiana Rossi ${ }^{1}$, Michio Seya ${ }^{1}$, and Tohn Takahashi ${ }^{1}$ \\ ${ }^{1}$ Integrated Support Center for Nuclear Nonproliferation and Nuclear Security, Japan Atomic Energy Agency, 2-4 Shirane Shirakata, \\ Tokai-mura, Ibaraki, JAPAN \\ ${ }^{2}$ Nuclear Security Unit, Joint Research Centre, Via E. Fermi 2749, I-21027 Ispra (VA), ITALY
}

\begin{abstract}
Safeguards verification of uranium and plutonium in high-radioactivity nuclear material is currently performed using destructive analysis techniques. However, the preparation method is a burden on both the safeguards inspectors and facility operators. While nondestructive assay (NDA) techniques would improve the efficiency and time, there are no passive NDA techniques available to directly verify the $\mathrm{U}$ and $\mathrm{Pu}$ content. As an alternative, the JAEA and JRC are collaboratively developing the Delayed Gamma-ray Spectroscopy (DGS) active-interrogation NDA technique to evaluate the fissile composition from the unique fission product yield distributions. To analyze the data we are developing an Inverse Monte Carlo (IMC) method that simulates the interrogation and evaluates the individual contributions from the mixed nuclear material to the composite spectrum. While the current nuclear data affects the ability to evaluate the composition, the IMC analysis method can be used to determine the systematic uncertainty contributions and has the potential to improve the nuclear data. We will present the current status of the DGS collaborative work as it relates to the development of the DGS IMC analysis.
\end{abstract}

\section{Introduction}

The objective of nuclear safeguards is to independently verify in a timely manner that civil nuclear material and activities are not diverted for the development of nuclear weapons. One vital element of this is for inspectors from the International Atomic Energy Agency (IAEA) to quantify the fissionable nuclides within nuclear material (NM) in order to verify facility operator declarations. For lowradioactivity NM (LRNM), passive nondestructive assay (NDA) measurement techniques are available. For instance in reprocessing plants, purified Pu-nitrate undergoes neutron coincidence counting (NCC) to quantify the total $\mathrm{Pu}$ mass by measuring the neutrons emitted during spontaneous fission. Using high-resolution gamma spectroscopy (HRGS), the isotopic composition is then determined by evaluating the ratio of their unique gamma rays emitted through $\alpha$ decay.

Comparatively, high-radioactivity NM (HRNM), like spent fuel solution in reprocessing plants, contains significant amounts of minor actinides and fission products (FPs) and present challenges for safeguards verification. Specifically, the neutrons emitted from curium make it impossible to directly evaluate the $\mathrm{Pu}$ and $\mathrm{U}$ of significant safeguard importance. Additionally, for every gamma ray (GR) emitted during a Pu decay, 100,000 GRs are emitted from fission products and measured with a GR detector. Consequently, there are no passive NDA techniques

*e-mail: rodriguez.douglaschase@jaea.go.jp currently available to quantify and verify HRNM. Instead IAEA inspectors utilize 1) the Hybrid K-Edge/X-ray Densitometry (HKED) technique to evaluate the elemental $\mathrm{Pu}$ and $U$ content and 2) Isotope Dilution Mass Spectrometry (IDMS) to provide a highly precise evaluation of the nuclide content. However, IDMS is a destructive analysis technique that has a time-consuming hot-cell preparation process that limits the number of verified samples to $20 \%$ of those measured with HKED and extends the reporting time to $\sim 10$ days [1] in addition to producing waste and consuming limited reference materials.

To address this challenge, the Japan Atomic Energy Agency (JAEA) and Joint Research Centre (JRC) of the European Commission are developing the delayed gamma-ray spectroscopy (DGS) technique to supplement the verification of HRNM [2]. DGS is an active interrogation technique where the NM is irradiate with neutrons to produce FPs that subsequently decay and release GRs, the quantification signature. Our primary goal is to develop a compact practical system that can be easily installed into an on-site laboratory of reprocessing plants. Toward this goal, joint measurements started in 2015 at JRC (see Figure 1), with the scope of evaluating an optimized system along with the spectral analysis method for determining the composition.

While other work describes the system development, this work focuses on the nuclear data used to develop the analysis of the measured spectra. First we describe the DGS technique itself and the JAEA-JRC system develop- 

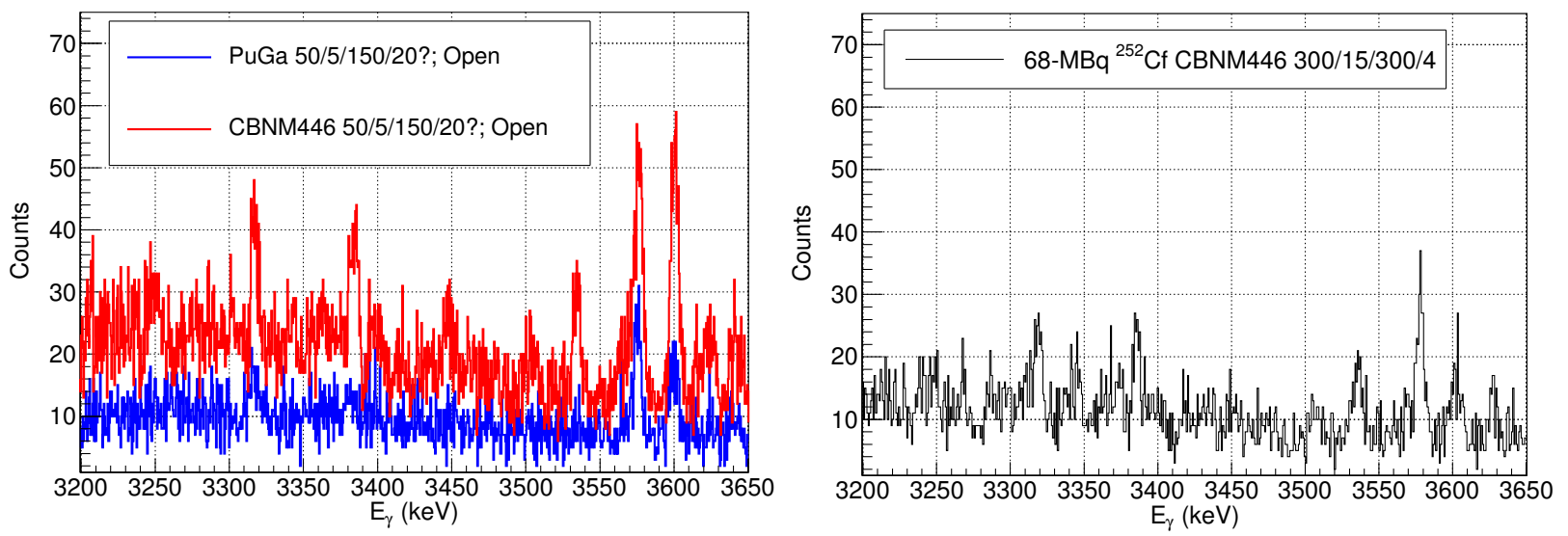

Figure 1: Measured spectra using PUNITA [3] (left) and a ${ }^{252} \mathrm{Cf}$ source in PERLA [4] (right).

ment. Following will be description of the DGS Monte Carlo and inverse Monte Carlo analysis presented in light of the technique. Specifically, we expand upon initial results [5] and explain how evaluated nuclear data affects both the analysis as well as system design.

\section{Delayed Gamma-ray Spectroscopy Development}

The DGS technique uses active interrogation to produce a signature of the fissionable content within a NM sample. The interrogation is a process that consists of an irradiation period, wherein the FPs are produced, and a measurement period, wherein the FPs decay and measured. For a given fission rate $(R)$, the number of nuclei $(N)$ of fission product $i$ are generated according to

$$
N_{i}=\left(1-e^{-\lambda_{i} t_{\text {Irrad }}}\right) R \sum_{j} Y_{i j} f_{j}
$$

where $\lambda_{i}$ is the FP decay constant, $t_{\text {Irrad }}$ is the irradiation time, and $Y_{i j}$ is the fission yield from the fissionable nuclide $j$ of molar fraction $f_{j}$ in the sample. After their production, these FP nuclei decay according to the equation

$$
N_{i}^{\prime}=N_{i}(0) e^{-\lambda_{i} t_{\text {decay }}}
$$

where $N(0)$ is the number of nuclei at the beginning of the decay period that is $t_{\text {decay }}$ long in time.

It is during the non-irradiation period that the GRs from the decaying FPs is measured to determine the composition, $f_{j}$. However, due to the intense low-energy passive GR emissions (e.g. $662-\mathrm{keV}$ from ${ }^{137} \mathrm{Cs}$ ) shielding materials are used to reduce their effects on the detection capability. As a consequence, this also limits the signature to those GRs from FPs that are emitted with high energy (HE), $E \gtrsim 3-\mathrm{MeV}$. Fortunately, most of the FPs producing GRs with these energies have short half-lives $(\tau \lesssim 10$ minutes), which is a benefit for fast verification improvements. To quantify the composition, the unique HE GRs must be distinguishable from the background using a combination of resolution and efficiency. Similar to the HRGS passive
NDA technique, DGS measurements are dominantly evaluated for high-purity germanium (HPGe) detectors that have very high resolution, $\sim 0.3 \%[6,7]$.

One primary goal of our JAEA-JRC development is to create a practical interrogation system that is compact and can be easily installed into an on-site laboratory. While the neutron source must then also be compact, this limits the options to deuterium-tritium (D-T) or deuteriumdeuterium (D-D) neutron generators or radioisotopes, like ${ }^{252} \mathrm{Cf}$ or AmBe sources. For these cases, the emission energy is either $\sim 2.5-$ or $14-\mathrm{MeV}$. In order to offset the required low-energy filtering, a moderator must be used to reduce the neutron energy to utilize the high fission crosssection of fissile nuclides (i.e. ${ }^{235} \mathrm{U},{ }^{239} \mathrm{Pu},{ }^{241} \mathrm{Pu}$ ) [8, 9].

However, using high-energy neutron sources can damage HPGe and other crystalline detectors. Consequently, our research includes determining the quantifiable difference between using $\mathrm{HPGe}$ and $\mathrm{LaBr}_{3}$ scintillator detectors. While $\mathrm{LaBr}_{3}$ has lower resolution than HPGe, it does not suffer from neutron damage. Additionally, the choice of detector also constrains the interrogation: using a standard HPGe detector requires it to be placed sufficiently far from the neutron source that subsequently adds a delay between the irradiation and measurement periods to transfer the irradiated sample. This transient (sample) configuration, though, may not be required with $\mathrm{LaBr}_{3}$ that could instead be placed in a static (sample) configuration to remove the delay.

\section{DGS Monte Carlo/Inverse Monte Carlo Analysis}

Compared to other aspects of the sample verification (e.g. number of samples, frequency), the DGS technique itself relies heavily upon nuclear data (ND) as seen in the previous section. Minimally, this shows in our evaluation of the optimum system design to produce a sufficient reaction rate ( $R$ in Equation 1). Beyond this, though, is that the production and decay rates of Equations 1 and 2 rely upon accurate and precise ND to predict and analyze the observed GR spectrum. As such, we are developing a Monte Carlo (MC) using ROOT [10] that utilizes multiple databases to 
Table 1: List of primary nuclear databases and codes used for the noted variable in our DGS Monte Carlo.

\begin{tabular}{ll}
\hline \multicolumn{1}{c}{ Database } & \multicolumn{1}{c}{ Variable } \\
\hline ENDF/B-VII.1 [11] & $\begin{array}{l}\text { Cross sections } \\
\text { Fission Yields }\end{array}$ \\
\hline \multirow{2}{*}{ ENSDF [12] } & $\begin{array}{l}\text { Half-lives } \\
\text { Branching Ratios } \\
\gamma \text { Intensity }\end{array}$ \\
\hline NIST [13] & Shielding \\
\hline MCNP [14] & Neutron Flux \\
\hline Geant4 [15] & Detector Efficiency \\
& Room Scatter \\
\hline
\end{tabular}

predict the expected spectrum (see Table 1). These spectra are evaluated for mono-nuclide samples in order to estimate the individual contributions from each nuclide that a single sample may contain (see Figure 2).

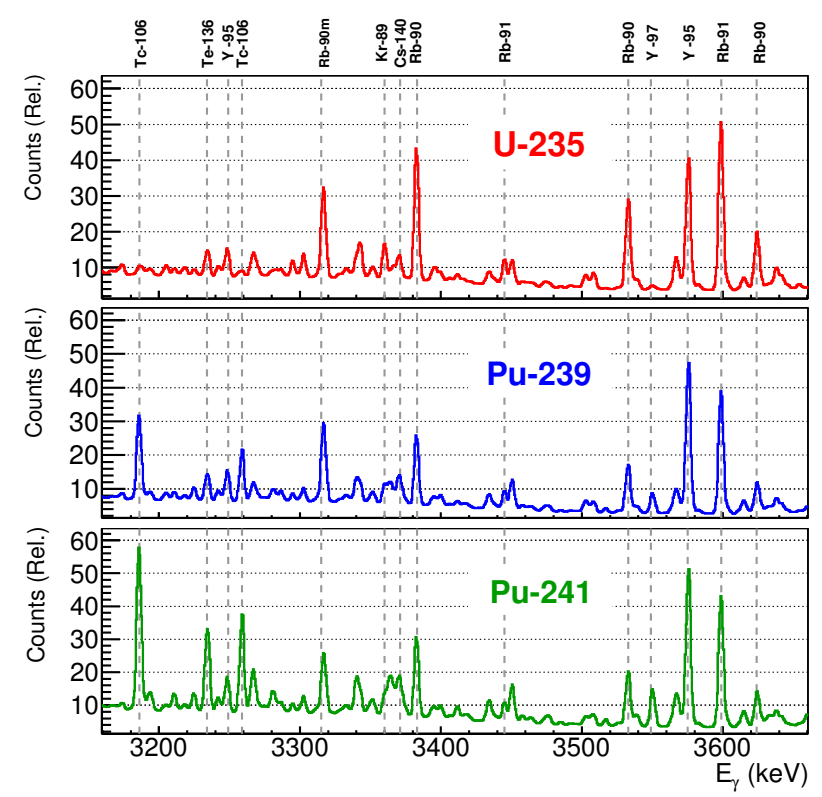

Figure 2: Comparison of noted simulated fissile nuclide high-energy gamma-ray spectra collected with an interrogation of 50-s irradiation, 150-s measurement, 5-s delay and reset, and 13 cycles.

The MC calculates each aspect of the interrogation, beginning with the energy dependent neutron flux $\left(\phi_{v}(E)\right)$ entering the empty sample space evaluated from the MCNP [14] model of the system. This is then scaled by possible neutron filters that surround the user-described cylindrical or rectilinear sample before interacting with sample material, both determined from cross-sections in nuclear databases: ENDF [11], JEFF [16], JENDL [17]. From this order of priority, the MC calculates the number of secondary nuclei produced from all known reaction modes, fission products ( $Y_{i j}$ in Equation 1) as well as activation products from the matrix materials. All sample nuclides are then scanned in a hierarchical method, starting with the primary parent that is not also a daughter product and decaying the unstable nuclides according to $N_{i}$ (Equation 2). Consequently, before a nuclide decays, the appropriate number of nuclei are added to the daughters determined from the branching ratios for all parents in the sample. The list of 4,217 nuclides (including metastable states), branching ratio, half-lives, and known decay GRs are derived from the ENSDF database [12] since it is updated regularly from recent data and theory.

This DGS MC allows for a delay $\left(t_{\text {Delay }}\right)$ between the irradiation period $\left(t_{\text {Irrad }}\right)$ and measurement period $\left(t_{\text {Meas }}\right)$, as well as an independent reset period $\left(t_{\text {Reset }}\right)$ to simulate the return of the sample to the irradiation position. The GRs emitted from the sample, including matrix materials that could interfere with the signature, are determined just during $t_{\text {Meas }}$ and factored into mono-energetic detector responses (e.g. escape peaks, Compton scatter, etc.) from Geant 4 simulations [15]. These are then scaled by user-described shielding materials defined from the NIST database [13] and distributed according to real detector calibration gains. These spectra for each cycle $(C)$ are then integrated and used as the simulated interrogation with options to retain both the nuclides per period and the GRs per cycle.

The GR peak-ratio analysis has multiple benefits derived from this DGSMC. First, the uncertainties of each ND database value can be used to quantify the variance of the composition evaluation ( $f_{j}$ in Equation 1). Randomly choosing a value within the database uncertainties and real-system characterization errors emulates the possible spread in the measured GR spectral peak ratios. This is extremely beneficial for HRNM since this uncertainty can be directly applied as the systematic uncertainty while limiting the number of repeatable interrogations for safety. Additionally, a sensitivity analysis can be performed to determine the dominant sources of the uncertainty and minimize their effects as possible. Present studies show that DGS is mostly affected by the interrogation timing through Equations 1 and 2 since the FP nuclei produced and the GRs observed during their decay directly changes the spectrum.

Most importantly, though, is that the resultant MC spectra of mono-nuclide samples using the mean database values can be used to evaluate the composition. This method, known as Inverse Monte Carlo (IMC), enables the individual nuclide expected spectra to be independently scaled to best match the resultant spectrum to result in the most-likely composition. Fitting the ${ }^{235} \mathrm{U}$ (CBNM446) spectrum interrogated with PUNITA from Figure 1 results in a $\sim 6 \%$ bias of $\mathrm{Pu}$. However, noting the two $\mathrm{Pu}$ isotopes in Figure 2 are extremely similar for this interrogation, ignoring the ${ }^{241} \mathrm{Pu}$ contribution results in much better agreement at a $\sim 0.5 \%$ bias of ${ }^{239} \mathrm{Pu}$.

Fitting simulated MC spectra similarly with the mean value expectation shows a composition variance of $\sim 1.8 \%$ for similar 50 -s/5-s/150-s $\times 13$-cycle ${ }^{235} \mathrm{U}$ samples, but a $\sim 16 \%$ uncertainty on mixed samples (see Figure $3 a$ ). However, changing the interrogation pattern to $10-\mathrm{s} / 1$ $\mathrm{s} / 10 \mathrm{~s} \times 50$ cycles improves the fit by $\sim 34 \%$, ignoring the fission yield uncertainty $\left(\sigma_{Y_{i j}}\right)$ drops the absolute variance to $\sim 2.6 \%$ (see Figure $3 b$ ). Since a pure ${ }^{235} \mathrm{U}$ set of sim- 


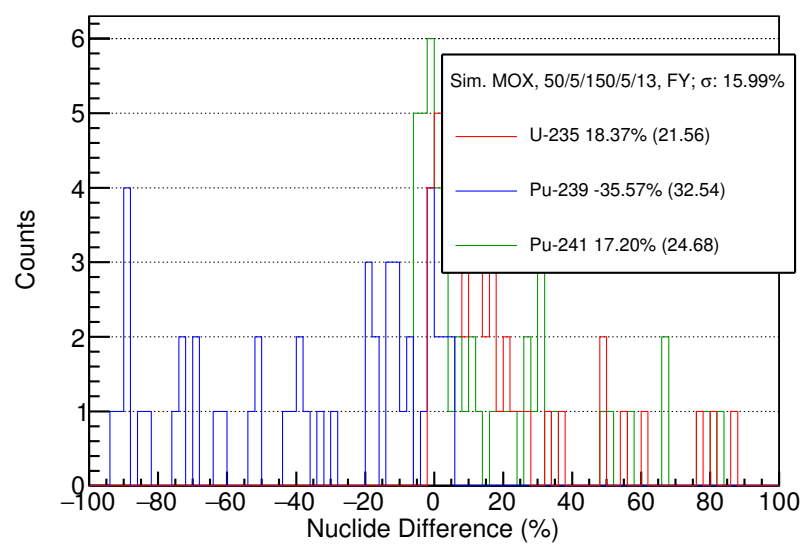

(a) High Variance using $\sigma_{Y_{i j}}$

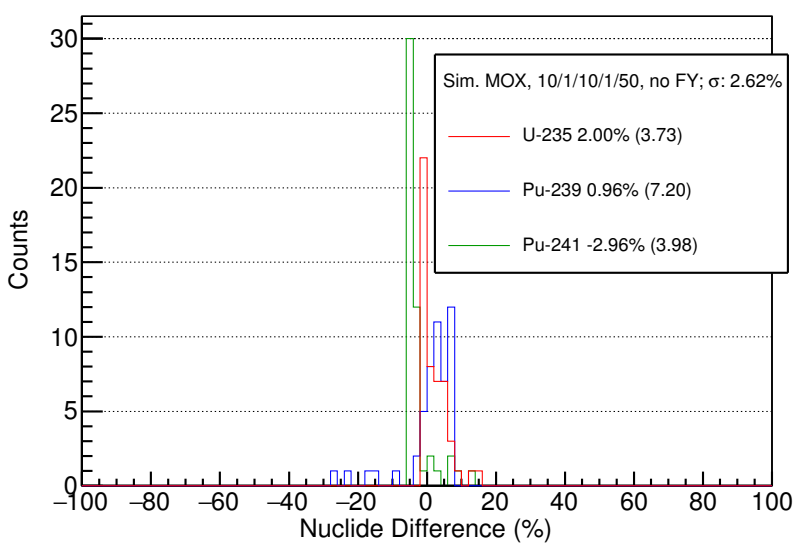

(b) Low Variance without $\sigma_{Y_{i j}}$

Figure 3: Average variance of mixed $\mathrm{U}$ and $\mathrm{Pu}$ material with the noted interrogation.

ulated spectral fits result in a $\sim 0.2 \%$ variance, it could be concluded that the mean $Y_{i j}$ values of the Pu isotopes may not be accurately known within the 30 to $70 \%$ uncertainties of the ENDF database. Comparing different databases, there is only a $\sim 3 \%$ variance on the composition evaluation based on ENDF, JEFF, and JENDL $Y_{i j}$ mean values. From this it must then be understood that the both the uncertainty and mean values of the Pu isotopes that are significant for composition evaluation of HRNM must be improved. Toward this end, additional measurements will be performed to improve the evaluation of mono-nuclide spectra based on interrogation patterns for absolute validation of the MC and improvements toward DGS safeguards.

\section{Summary}

The JAEA and JRC are collaborating on the development of a practical delayed gamma-ray spectroscopy system for safeguards verification of high-radioactivity nuclear material. Measured spectra for analysis development shows that DGS is sensitive to the particular interrogation performed on the sample. These measurements are being used to validate the Monte Carlo used for both the simulation and analysis and is showing a large variance due to the fission yield uncertainties in the nuclear databases. Future measurements will be performed to improve our DGS capability for direct application to safeguards.

\section{Acknowledgements}

This work was partially supported by subsidy to promote strengthening nuclear security related activities from the Japanese Ministry of Education, Culture, Sports, Science, and Technology.

\section{References}

[1] T. Itoh, S. Hara, Y. Sato, Y. Itoh, T. Sawahata, K. Naito, T. Hayakawa, G. Duhamel, Tech. rep., International Atomic Energy Agency, Vienna (Austria) (2010)
[2] D.C. Rodriguez, F. Rossi, T. Takahashi, M. Seya, M. Koizumi, J.M. Crochemore, G. Varasano, T. Bogucarska, K. Abbas, B. Pedersen, Development of Delayed Gamma-ray Spectroscopy for Nuclear Material Analysis, in 59th Annual Meeting of the Institute of Nuclear Materials Management (2018)

[3] H. Rennhofer, B. Pedersen, J.M. Crochemore, Neutron flux from a 14-MeV neutron generator with tungsten filter for research in NDA methods for nuclear safeguards and security, in American Institute of Physics Conference Series, edited by K. BaharuthRam (2009), Vol. 1194 of American Institute of Physics Conference Series, pp. 36-42

[4] V. Esteban Gran, Tech. rep., Joint Research Centre, Brussels (Belgium) (2015)

[5] D.C. Rodriguez, F. Rossi, M. Seya, M. Koizumi, Delayed Gamma-Ray Spectroscopy Inverse Monte Carlo Analysis Method for Nuclear Safeguards Nondestructive Assay Applications, in IEEE Nuclear Science Symposium and Medical Imaging Conference (2017), p. Paper 3003

[6] L. Campbell, A. Hunt, B. Ludewigt, V. Mozin, Journal of Nuclear Materials Management 40, 78 (2012)

[7] D.C. Rodriguez, E. Anderson, K.K. Anderson, L.W. Campbell, J.E. Fast, K. Jarman, J. Kulisek, C.R. Orton, R.C. Runkle, S. Stave, Applied Radiation and Isotopes 82, 181 (2013)

[8] D.C. Rodriguez, F. Rossi, T. Takahashi, M. Seya, M. Koizumi, Applied Radiation and Isotopes (2019)

[9] F. Rossi, D.C. Rodriguez, T. Takahashi, M. Seya, M. Koizumi, Delayed Gamma-ray Spectroscopy for Nuclear Material Analysis (2): A Practical System with DD Neutron Generator, in 60th Annual Meeting of the Institute of Nuclear Materials Management (2019)

[10] R. Brun, F. Rademakers, Nuclear Instruments and Methods in Physics Research Section A: Accelerators, Spectrometers, Detectors and Associated Equipment 389, 81 (1997) 
[11] M.B. Chadwick et al., Nucl. Data Sheets 112, 2887 (2011)

[12] J.K. Tuli, Tech. rep., Brookhaven National Laboratory (2001)

[13] J.H. Hubbell, S.M. Seltzer, Tables of X-Ray Mass Attenuation Coefficients and Mass EnergyAbsorption Coefficients (version 1.4) [Online] Available: (2014), https://www.nist.gov/pml/ $\mathrm{x}$-ray-mass-attenuation-coefficients

[14] T. Goorley, et al., Nuclear Technology 180, 298 (2012)

[15] S. Agostinelli, J. Allison, K. Amako, J. Apostolakis, H. Araujo, P. Arce, M. Asai, D. Axen, S. Banerjee, G. Barrand et al., Nuclear Instruments and Methods in Physics Research Section A: Accelerators, Spectrometers, Detectors and Associated Equipment 506, 250 (2003)

[16] A. Koning, M. Avrigeanu, V. Avrigeanu, P. Batistoni, E. Bauge, M.M. B'e, P. Bem, D. Bernard, O. Bersillon, A. Bidaud et al., The JEFF evaluated nuclear data project, in International Conference on Nuclear Data for Science and Technology (2007), https: //www. oecd-nea.org/dbdata/jeff/

[17] K. SHIBATA, O. IWAMOTO, T. NAKAGAWA, N. IWAMOTO, A. ICHIHARA, S. KUNIEDA, S. CHIBA, K. FURUTAKA, N. OTUKA, T. OHSAWA et al., Journal of Nuclear Science and Technology 48, 1 (2011), https://doi.org/10.1080/18811248.2011.9711675 